\title{
miR-146a and miR-146b in the diagnosis and prognosis of papillary thyroid carcinoma
}

\author{
ZHENWEI QIU $^{1 *}$, HAI LI ${ }^{1 *}$, JIANWEI WANG ${ }^{1}$ and CHONGBING SUN ${ }^{2}$ \\ ${ }^{1}$ Department of General Surgery, Yidu Central Hospital of Weifang, Weifang, Shandong 262500; \\ ${ }^{2}$ Department of General Surgery, Weifang People's Hospital, Weifang, Shandong 261041, P.R. China
}

Received April 26, 2017; Accepted September 13, 2017

DOI: 10.3892/or.2017.5994

\begin{abstract}
The present study investigated the relationship between the expression of miR-146a and miR-146b with the occurrence and prognosis of papillary thyroid carcinoma. Experiments in vitro were also used to explore the effect of the knocked down expression of the miRNAs on growth and migration of papillary thyroid carcinoma cells. A total of 73 patients with papillary thyroid carcinoma admitted to Yidu Central Hospital of Weifang from September 2013 to September 2015 were enrolled in the study. Carcinoma samples were obtained from each patient, and adjacent tissues were used as control samples to determine expression levels of miR-146a and miR146b by semi-quantitative RT-PCR. An analysis was conducted to find possible correlations between the miRNAs expression levels and clinicopathological features in the patients followed up for one year after diagnosis. Additionally, to examine the function of miR-146a and miR-146b on TPC-1 cells, the expression of miRNAs was knocked down using specific siRNAs. MTT and Transwell assays were used to evaluate cell proliferation and migration, respectively, in the miRNA cell lines. Finally, western blot analysis was used to analyze the expression of IRAK1 in PTC cancer cells. Our results showed that the expression levels of miR-146a and miR-146b in carcinoma tissues were significantly higher than the levels in cancer-free tissues $(\mathrm{P}<0.01)$. The relative expression levels of miR-146a and miR-146b in cancerous tissues could be associated with the pathological type and presence or absence of lymph node metastasis $(\mathrm{P}<0.05)$. Compared with the siRNA-control cell, MTT and Transwell assays showed that the cell growth and migration of TPC- 1 cells were decreased in miR-146a and miR-146b low expression cells $(\mathrm{P}<0.01)$. Western blot analysis showed that
\end{abstract}

Correspondence to: Dr Chongbing Sun, Department of General Surgery, Weifang People's Hospital, 151 Guangwen Street, Kuiwen, Weifang, Shandong 261041, P.R. China

E-mail: chongbing_sun@163.com

${ }^{*}$ Contributed equally

Key words: miR-146a, miR-146b, papillary thyroid carcinoma, prognosis the expression of IRAK1 in papillary thyroid carcinoma was higher than in adjacent tissue $(\mathrm{P}<0.01)$. Based on our findings, the expression of miR-146a and miR-146b correlates with the occurrence and prognosis of papillary thyroid carcinoma, and the expression levels of miR-146a and miR-146b seem to affect the cell proliferation and migration and regulate the expression of IRAK1 protein in cancer cells. Further studies are needed to validate our results to provide new targets for prevention and treatment of papillary thyroid carcinoma.

\section{Introduction}

The development of thyroid cancer is closely related to a variety of factors, papillary thyroid carcinoma (PTC) ranks highest in incidence amongst thyroid cancers; epidemiological surveys have shown that the incidence of PTC has a rising trend, and that it occurs more frequently in women (1-3). Even with recent advancements in medical technology, PTC is difficult to diagnose at an early stage because of its insidious onset devoid of clinical symptoms, leading to its poor prognosis at the time of diagnosis.

A common treatment for PTC includes surgical resection followed by radioactive ${ }^{131} \mathrm{I}$ therapy and other chemotherapeutic drugs (4-6). Molecular biology research has made microRNAs (miRNAs) a hotspot in the medical and life sciences, and a large number of studies have shown that miRNAs are closely related to the development and progression of multiple cancers $(7,8)$. A recent study found that the overexpression of miR-146a can significantly increase the incidence of ovarian cancer, and that clinical treatment with a drug blocking miR-146a expression can significantly increase the survival time of patients (9). Also, the expression of miR-146b is associated with the occurrence of breast cancer, and is closely related to the proliferation and migration of breast cancer cells (10).

The IRAK family proteins participate in the TLRs/IL-1 signaling pathway, that is involved in intracellular signal control and the inflammatory response. The IRAK1 protein is closely related to the development of inflammation and tumors. However, there are no reports on the relationship between the expression of miR-146a and miR-146b in PTC and whether IRAK1 protein is involved in their regulation.

In this study, the expression levels of miR-146a and miR-146b in PTC tissues were quantified, and statistical analyses were performed looking for possible associations to 
Table I. PCR primers.

\begin{tabular}{ll}
\hline Gene & \multicolumn{1}{c}{ Sequence } \\
\hline miR-146a & F: 5'-ATCCACCTTGACGATGCTTTAC-3' \\
& R: 5'-TTCAGATGTTCTAAGCCTACGG-3' \\
miR-146b & F: 5'-TGGCCCTCGTAGCCTTGAGGAC-3' \\
& R: 5'-CCAGTGCTGCAGGGTCCGAGGT-3' \\
$\beta$-actin & F: 5'-GATGATTGGCATGGCTTT-3' \\
& R: 5'-CACCTTCCGTTCCAGTTT-3'
\end{tabular}

F, forward; R, reverse.

clinical and pathological features and prognosis of patients. Additionally, siRNAs were used to transfect TPC-1 cells in order to knock down their expression levels of miR-146a and miR-146b and compare their proliferation and migration abilities.

\section{Materials and methods}

Subjects and biological samples. The samples (73 in total) in this study were selected from PTC tumors removed by surgery and confirmed by pathology from patients admitted to Yidu Central Hospital of Weifang from September 2013 to September 2015. The patients' ages ranged from 38 to 67 years, there were 34 males and 39 females; all patients were cleared from other severe diseases and all signed informed consent forms. All patients presented complete clinical and pathological data. Cancer-free tumor adjacent tissue samples (at least $5 \mathrm{~cm}$ away from tumor) were also examined as negative controls. All patients underwent standard treatment and were followed up for 1-year after diagnosis. Samples of cancer and adjacent tissue from each patient were stored in liquid nitrogen. The study was approved by the Ethics Committee of Yidu Central Hospital of Weifang.

Semi-quantitative RT-PCR detection of the expression of miR-146a and miR-146b in cancer tissues. Tissue samples were taken out of the liquid nitrogen storage and thawed. The total RNA was extracted from each sample according to the instructions on the TRIzol kit (Invitrogen, Carlsbad, CA, USA). The quality of the resulting RNA samples was confirmed by agarose gel electrophoresis. The gels showed clear and distinct 28S, $18 \mathrm{~S}$ and $5 \mathrm{~S}$ bands with the brightness of the $28 \mathrm{~S}$ band being about twice that of the $18 \mathrm{~S}$, indicating that the RNA was intact and could be used for subsequent experiments. Then, cDNA was produced using a reverse transcription kit (Invitrogen), and the expression levels of miR-146a and miR-146b in cancer and adjacent tissues were detected by semi-quantitative PCR using a SYBR ExScript ${ }^{\mathrm{TM}}$ RT-PCR kit (Takara Bio, Shiga, Japan), using $\beta$-actin as internal reference gene. The PCR reaction conditions included 35 cycles of $95^{\circ} \mathrm{C}$ for $30 \mathrm{sec}$ for denaturation, $64^{\circ} \mathrm{C}$ for $25 \mathrm{sec}$ for annealing, and $72^{\circ} \mathrm{C}$ for $30 \mathrm{sec}$ for extension. Primers were synthesized by Tiangen Biotech Co., Ltd. (Beijing, China) and the sequences are shown in Table I. After the amplification, agarose gel elec- trophoresis was performed and the gel was observed using an UV imaging system. Each RT-PCR experiment was repeated at least three times for statistical analysis.

Construction of miR-146a and miR-146b low expression cell lines.miR146a-siRNA, miR146b-siRNA and its corresponding control sequences were designed and synthesized by Toyobo Co., Ltd. (Osaka, Japan). Proliferating TPC-1 cells (Chinese Academy of Sciences Shanghai Cell Bank) were transfected with the synthesized siRNAs. After $48 \mathrm{~h}$, total RNA was extracted from the cells and cDNA was obtained by reverse transcription, the expression of miR-146a and miR-146b was detected by semi-quantitative RT-PCR. Successfully transfected cells were placed in incubator and cultured at $37^{\circ} \mathrm{C}$, $5 \% \mathrm{CO}_{2}$ for subsequent experiments. The expression levels of miR-146a and miR-146b were low in the miRNA-transfected cells but not so in the ones transfected with negative control siRNAs.

Detection of the effect of low expression of miR-146a and miR-146b on TPC-1 cells

MTT assays for proliferation of thyroid papillary carcinoma cells. The MTT assay was used to compare the proliferation of the different siRNA-transfected TPC-1 cells. MTT (SigmaAldrich, St. Louis, MO, USA) was prepared at $5 \mathrm{mg} / \mathrm{ml}$. Ninety-six-well plates with $3 \times 10^{4}$ cells/well were incubated for $48 \mathrm{~h}$ before adding MTT. Then, after $4 \mathrm{~h}$ of incubation, the medium was discarded. Dimethyl sulfoxide (DMSO) (Sigma-Aldrich) was added to each well and the absorbance values were measured at $570 \mathrm{~nm}$ using a microplate reader (Eppendorf, Hamburg, Germany).

Transwell migration assays for transfected papillary thyroid carcinoma cells. Transwell assays were performed to investigate the effect of low expression of miR-146a and miR-146b on the migration of papillary thyroid carcinoma cells. After 24-h starvation, the cells were adjusted to a concentration of $5 \times 10^{5}$ cells $/ \mathrm{ml}$ and added to the Transwell chamber. The number of cells passing through the chamber was calculated under a microscope (Olympus, Tokyo, Japan) after dyeing and fixing (10).

Detection of the expression level of IRAK1 protein by western blot analysis. The samples from the papillary thyroid carcinoma tissues and adjacent healthy tissues were taken out of the liquid nitrogen. The tissue was cut with scissors, and the cells were homogenized in lysis buffer. After centrifugation $2,600 \mathrm{x} \mathrm{g}$ for $5 \mathrm{~min}$, the supernatant was transferred to a fresh tube to save the soluble protein. A Protein Quantification kit (Millipore, Billerica, MA, USA) was used to quantify the extracted protein, and the protein samples of similar concentration were prepared. The samples were loaded onto SDS-PAGE and then transferred to a western blot membrane. After a standard transfer, the membranes were blocked and washed before adding the rabbit anti-human IRAK1 polyantibody (dilution, 1:1,000; cat. no. 06-872; Millipore) for overnight incubation at $4^{\circ} \mathrm{C}$. Next morning, the membranes were washed 3 times with TBST before adding the goat anti-rabbit peroxidase polyantibody (dilution, 1:5,000; cat. no. A0545; Sigma-Aldrich, St. Louis, MO, USA) and incubating at room temperature for $2 \mathrm{~h}$. 
A

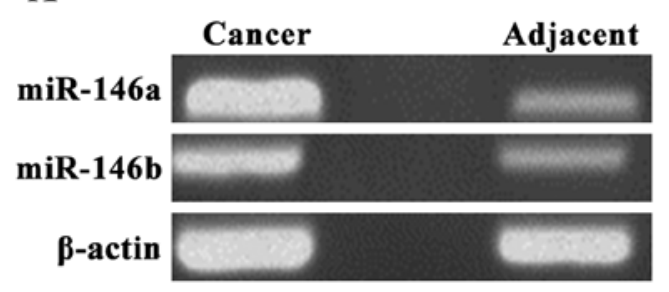

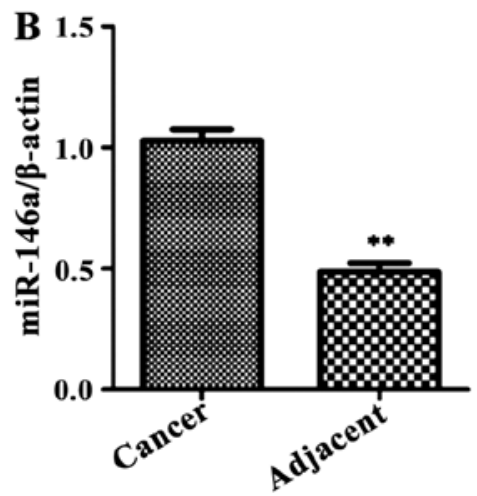

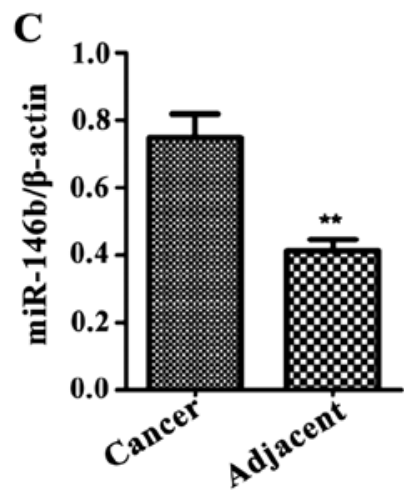

Figure 1. Relative expressions of miR-146a and miR-146b in cancer and adjacent tissues of papillary thyroid cancer patients by semi-quantitative RT-PCR. (A) Agarose gel electrophoresis; (B and C) relative expression levels of miR-146a and miR-146b, the relative expression of miR-146a and miR-146b in cancer tissues was significantly higher than in the adjacent tissues in papillary thyroid carcinoma patients $\left({ }^{* *} \mathrm{P}<0.01\right)$.

Table II. Relationship between the expression of miR-146a and miR-146b and different clinical features of PTC patients.

\begin{tabular}{|c|c|c|c|c|c|}
\hline Item & $\mathrm{n}$ & miR-146a expression & P-value & miR-146b expression & P-value \\
\hline Age (years) & & & 0.0692 & & 0.0782 \\
\hline$\leq 50$ & 37 & $5.98 \pm 1.33$ & & $3.53 \pm 1.08$ & \\
\hline$<50$ & 36 & $6.16 \pm 1.28$ & & $3.85 \pm 1.12$ & \\
\hline GA125 (U/ml) & & & 0.0619 & & 0.0873 \\
\hline$\leq 35$ & 43 & $5.87 \pm 1.08$ & & $5.63 \pm 1.62$ & \\
\hline$>35$ & 30 & $6.16 \pm 1.22$ & & $5.57 \pm 1.29$ & \\
\hline FIGO staging & & & 0.0538 & & 0.0686 \\
\hline Stage I-II & 28 & $6.29 \pm 1.39$ & & $6.08 \pm 1.19$ & \\
\hline Stage III-IV & 45 & $6.89 \pm 1.87$ & & $5.95 \pm 2.01$ & \\
\hline Lymphatic metastasis & & & 0.0078 & & 0.0097 \\
\hline Yes & 36 & $6.76 \pm 2.01$ & & $6.73 \pm 1.78$ & \\
\hline No & 37 & $3.58 \pm 1.96$ & & $2.17 \pm 1.05$ & \\
\hline Recurrence & & & 0.0086 & & 0.0176 \\
\hline Yes & 17 & $5.97 \pm 1.86$ & & $5.32 \pm 1.25$ & \\
\hline No & 56 & $2.38 \pm 1.29$ & & $3.17 \pm 1.03$ & \\
\hline
\end{tabular}

The membranes were then triple washed with TBST. Finally, the chemiluminescent signals were visualized and the target protein bands were scanned for analysis. The expression of $\beta$-actin was used as an internal reference (rabbit anti-human monoantibody; 1:5,000; cat. no. A5060; Sigma-Aldrich); the sheep serum for blocking from Jackson ImmunoResearch Laboratories, Inc. (West Grove, PA, USA).

Statistical analysis. The data in this study were expressed as mean \pm standard deviation values. The SPSS 19.0 software (SPSS, Inc., Chicago, IL, USA) was used for statistical analyses. The measurement data were analyzed using the t-test. Comparisons between groups of enumeration data were analyzed by the $\chi^{2}$ test. The homogeneity of variance test was performed, if the variance was homogeneous, the comparison between two was conducted using the Bonferronic method, if the variance was not homogeneous, then the Welch's method was adopted. Additionally, the Dunnett's T3 method was used for multiple comparisons, and the Pearson correlation statis- tical analysis for correlation analysis. A $\mathrm{P}<0.05$ for any given difference was considered as statistically significant.

\section{Results}

miR-146a and miR-146b expression levels in PTC patients. The expression levels of miR-146a and miR-146b in cancer and adjacent healthy tissues of PTC patients (73 samples each) were detected by semi-quantitative RT-PCR. The results showed that the relative expression of miR-146a and miR-146b in cancer tissues was significantly higher than in the healthy tissues $(\mathrm{P}<0.01)$, as shown in Fig. 1.

Relationship between the expression levels of miR-146a and $m i R-146 b$ and the clinicopathological features and prognosis of patients. The expression levels of miR-146a and miR-146b in the tissues of papillary thyroid carcinoma detected by ELISA were recorded to find any possible correlations with patient's age, tumor size, clinical stage or other 


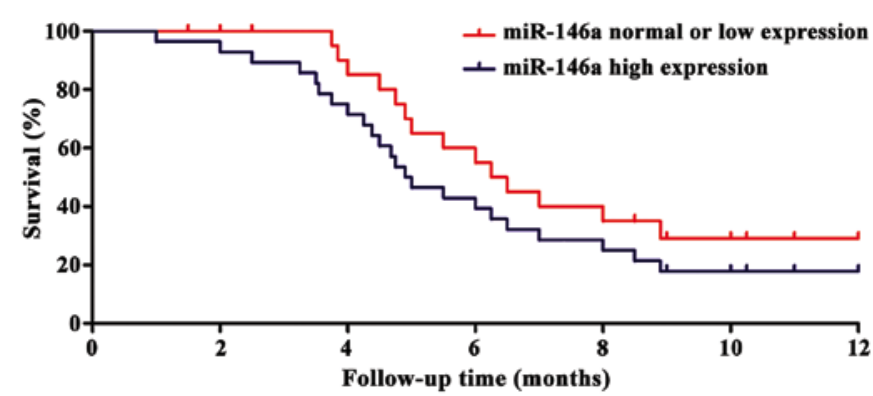

Figure 2. Relationship between the expression of miR-146a and the survival time. The survival time of the high miR-146a expression group was significantly shorter than that of the normal or low miR-146a expression groups, $(\mathrm{P}<0.01)$.

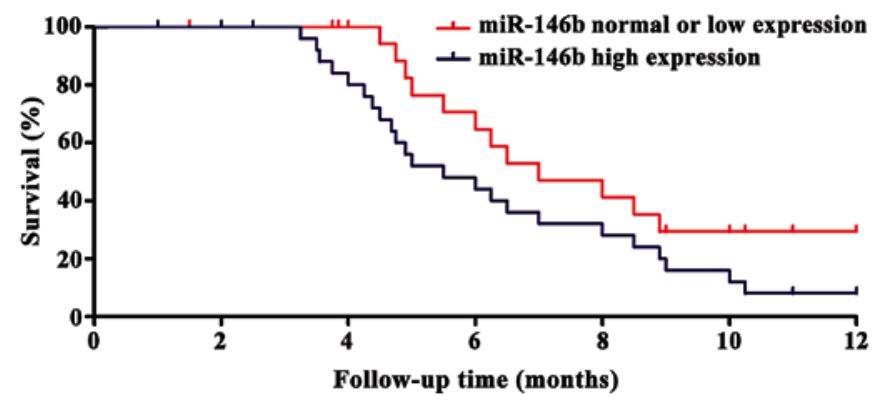

Figure 3. Relationship between the expression of miR-146b and the surviva time. The survival time of high miR-146b expression group was significantly shorter than that of the normal or low miR-146b expression groups $(\mathrm{P}<0.01)$.

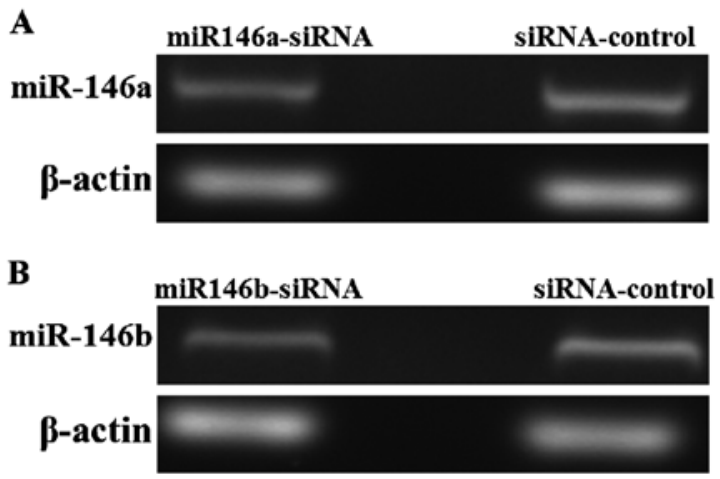

Figure 4. Semi-quantitative RT-PCR to verify expression levels of miR-146a and miR-146b in TPC-1 cell lines. (A) miR-146a expression; (B) miR-146b expression.

clinical features, and the results are shown on Table II. The relative expression of miR-146a and miR-146b in peripheral blood of PTC patients does not correlate with age, GA125 or FIGO staging $(\mathrm{P}>0.05)$. However, the relative expression correlates with the presence of lymph node metastasis and cancer recurrence $(\mathrm{P}<0.05)$. The expression levels were used to classify the data in groups with either high, normal or low expression levels of miR-146a and miR-146b. During the follow-up year, it was observed that the survival time of the patients with high expression of miR-146a and miR-146b was significantly shorter than that of the patients in the normal or low expression groups $(\mathrm{P}<0.01)$. The survival curves can be seen in Figs. 2 and 3.
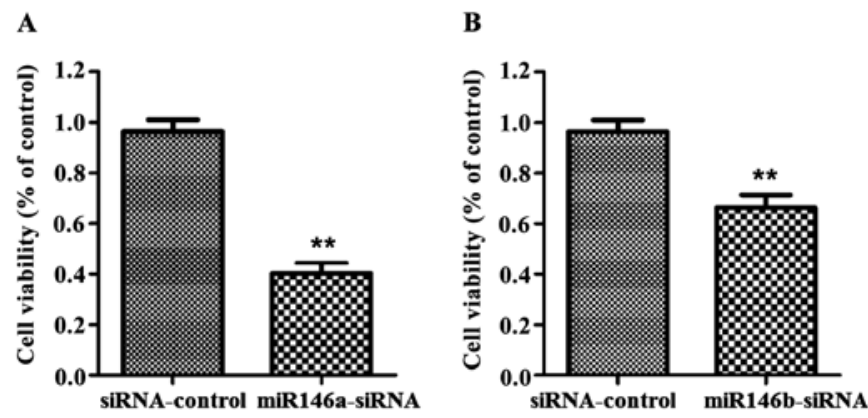

Figure 5. Cell proliferation detected by MTT. (A) Effect of low expression of miR-146a on cell proliferation, the number of cells in the miR146asiRNA group was significantly lower than that in the siRNA-control group $\left({ }^{* *} \mathrm{P}<0.01\right)$. (B) Effect of low expression of miR-146b on cell proliferation, the number of cells in the miR146b-siRNA group was significantly lower than that in the siRNA-control group $\left({ }^{* *} \mathrm{P}<0.01\right)$.
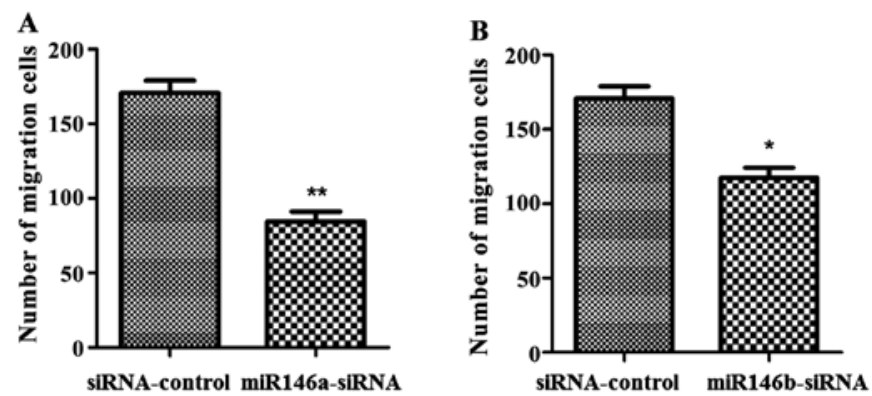

Figure 6. Cell migration ability detected by Transwell assay. (A) Effect of miR-146b knock-down on the migration of TPC-1 cells; (B) effect of miR-146b knock-down on the migration of TPC-1 cells $\left({ }^{*} \mathrm{P}<0.05,{ }^{* *} \mathrm{P}<0.01\right)$.

Construction of miR-146a and miR-146b low expression TPC-1 cell lines. The expression of miR-146a and miR-146b in thyroid papillary carcinoma cells TPC-1 were downregulated using a standard siRNA method. Next, total RNA was extracted and the expression was detected with semiquantitative PCR after reverse transcription. The results are shown in Fig. 4. Compared with the cells transfected with siRNA-control, the expression of miR-146a in the miR146asiRNA transfected cells decreased to $17.82 \pm 3.42 \%(\mathrm{P}<0.01)$. The expression of miR-146b in the miR146b-siRNA cells decreased $23.67 \pm 5.83 \%(\mathrm{P}<0.01)$, indicating that the miR-146a and miR-146b knock-down TPC-1 cell lines were successfully constructed.

Effects of low expression of $m i R-146 a$ and $m i R-146 b$ on cell proliferation. The MTT assay was used to determine proliferation of the knock-down cell lines, as shown in Fig. 5. The numbers of cells in the miR146a-siRNA and the miR146bsiRNA group were significantly lower than the number of cells in the siRNA-control group $(\mathrm{P}<0.01)$.

Effects of low expression of miR-146a and miR-146b on cell migration. The Transwell assay was used to assess cell migration on the miR-146a and miR-146b knock-down cell lines, the results are shown in Fig. 6. The migration ability of cells in the miR146a-siRNA and miR146b-siRNA groups was significantly lower than the migration ability of cells in the siRNA-control group. 

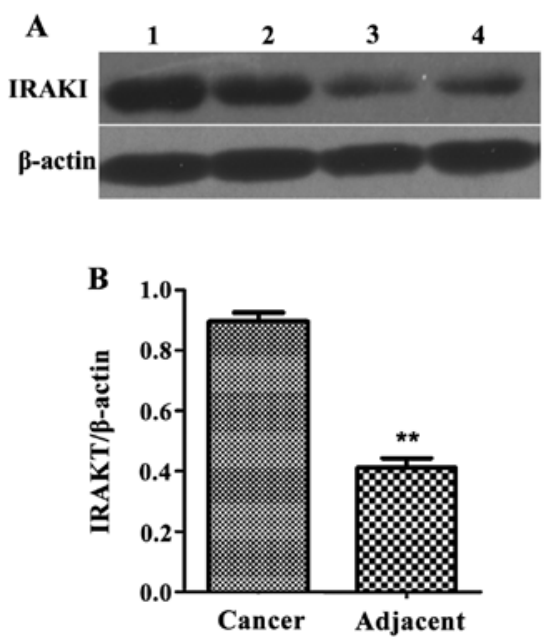

Figure 7. Western blot detection of IRAKI protein expression levels. (A) Western blot analysis, lanes 1 and 2 show papillary thyroid carcinoma samples; lanes 3 and 4 show the coresponding adjacent tissues. (B) Relative expressions of IRAK1 protein, compared with the adjacent tissues, the expression level of IRAKI protein in the cancer tissues of papillary thyroid carcinoma was significantly decreased $\left({ }^{* *} \mathrm{P}<0.01\right)$.

Detection of the expression of IRAK1 protein by western blot analysis. In order to investigate the effect of miR-146a and miR-146b on the expression of IRAK1 protein, the expression levels of IRAK1 protein in cancer and healthy adjacent tissues of patients with papillary thyroid carcinoma was detected by western blot analysis. The results are shown in Fig. 7. Compared with the normal tissues, the expression level of IRAK1 protein in the cancer tissues was significantly decreased $(\mathrm{P}<0.01)$.

\section{Discussion}

The regulation of miRNAs on human pathological and physiological processes is done in the body mainly through the expression of proteins during and after gene transcription. miRNAs can induce abnormal metabolism of tumor cells by influencing their transcription and translation processes $(11,12)$. High expression of miRNAs causing abnormal proliferation and migration of tumor cells can induce cancer, however in other cases the role of miRNAs can be tumor suppression (13). In studies of miRNAs, it has been observed that miR-146a expression is significantly higher in a variety of tumor cells than in normal tissues, and miR-146a is mostly involved in inflammation (14-16). A study found that breast cancer cells expressing high levels of miR-146a were more prone to distant metastasis than tumor cells with low expression levels of miR-146a (17). Other researchers using an animal sarcoma model also found that miR-146a can affect the proliferation and migration of tumor cells, and that proliferation and migration were significantly decreased after knockout of miR-146a (18). At present, there are relatively few studies on miR-146b. A research group found evidence for a role of miR-146b on the pathogenesis of colon cancer, where the possibility of colon cancer in miR-146b overexpression patients is significantly increased (19).

In this study, it was found that the expression levels of miR-146a and miR-146b were closely related to the occurrence of PTC. This indicates that regulating the expression of miR146a and miR-146b may be a valid therapeutic strategy against cancer. The prognoses of patients with normal or low expression of miR-146a and miR-146b were significantly better than those of patients with high expression levels. The study of the relationship between the expression of miR-146a and miR-146b and PTC clinicopathological features showed that the expressions of miR-146a and miR-146b was closely related to the metastasis and recurrence of PTC $(\mathrm{P}<0.01)$. miR-146a and miR-146b may lead to PTC recurrence and metastasis by affecting the proliferation and migration ability of papillary thyroid cancer cells. At present, there is evidence that miR$146 \mathrm{a}$ and miR-146b are mainly involved in inflammation processes (19). Therefore, the regulation of inflammation by these two genes may also affect the progress of PTC. Western blot analysis showed that miR-146a and miR-146b could significantly increase the expression of IRAK1. Other researchers found that $\mathrm{miR}-146 \mathrm{~b}$ can upregulate IRAK1 expression and plays a role in the inflammatory response in ovarian cancer (20). However, the mechanisms by which miR-146a and miR-146b regulate the expression of IRAK1 protein and their effects on the occurrence and prognosis of PTC is not clear at present.

In summary, miR-146a and miR-146b were highly expressed in PTC cancer tissues; and the prognoses of patients were related to the levels of expression of both miRNAs in cancer cells. The relative expressions of miR-146a and miR-146b should be useful as molecular markers for PTC screening, and the miRNAs could provide new targets for the clinical treatment against PTC.

\section{References}

1. Lai XJ, Zhang B, Jiang YX, Li JC, Zhao RN, Yang X, Zhang Q, Zhang XY, Li WB and Zhu SL: High risk of lateral nodal metastasis in lateral solitary solid papillary thyroid cancer. Ultrasound Med Biol 42: 75-81, 2016.

2. Liu TR, Su X, Qiu WS, Chen WC, Men QQ, Zou L, Li ZQ, Fu XY and Yang AK: Thyroid-stimulating hormone receptor affects metastasis and prognosis in papillary thyroid carcinoma. Eur Rev Med Pharmacol Sci 20: 3582-3591, 2016.

3. Wojakowska A, Chekan M, Marczak Ł, Polanski K, Lange D, Pietrowska M and Widlak P: Detection of metabolites discriminating subtypes of thyroid cancer: Molecular profiling of FFPE samples using the GC/MS approach. Mol Cell Endocrinol 417: 149-157, 2015.

4. Tan A, Stewart CJ, Garrett KL, Rye M and Cohen PA: Novel BRAF and KRAS mutations in papillary thyroid carcinoma arising in struma ovarii. Endocr Pathol 26: 296-301, 2015.

5. Soylu L, Aydin OU, Ozbas S, Bilezikci B, Ilgan S, Gursoy A and Kocak S: The impact of the multifocality and subtypes of papillary thyroid carcinoma on central compartment lymph node metastasis. Eur Rev Med Pharmacol Sci 20: 3972-3979, 2016.

6. Kamaya A, Tahvildari AM, Patel BN, Willmann JK, Jeffrey RB and Desser TS: Sonographic detection of extracapsular extension in papillary thyroid cancer. J Ultrasound Med 34: 285-297, 2015

7. Li B, Yang XX, Wang D and Ji HK: MicroRNA-138 inhibits proliferation of cervical cancer cells by targeting c-Met. Eur Rev Med Pharmacol Sci 20: 1109-1114, 2016.

8. Shu XL, Fan CB, Long B, Zhou X and Wang Y: The anti-cancer effects of cisplatin on hepatic cancer are associated with modulation of miRNA-21 and miRNA-122 expression. Eur Rev Med Pharmacol Sci 20: 4459-4465, 2016.

9. Sharma N, Verma R, Kumawat KL, Basu A and Singh SK: miR-146a suppresses cellular immune response during Japanese encephalitis virus JaOArS982 strain infection in human microglial cells. J Neuroinflammation 12: 30, 2015. 
10. Al-Ansari MM and Aboussekhra A: miR-146b-5p mediates p16-dependent repression of IL- 6 and suppresses paracrine procarcinogenic effects of breast stromal fibroblasts. Oncotarget 6: 30006-30016, 2015.

11. Rafiee-Pour HA, Behpour M and Keshavarz M: A novel labelfree electrochemical miRNA biosensor using methylene blue as redox indicator: Application to breast cancer biomarker miRNA-21. Biosens Bioelectron 77: 202-207, 2016.

12. Esposito CL, Catuogno S and de Franciscis V: Aptamer-MiRNA conjugates for cancer cell-targeted delivery. Methods Mol Biol 1364: 197-208, 2016.

13. Williams J, Smith F, Kumar S, Vijayan M and Reddy PH: Are microRNAs true sensors of ageing and cellular senescence? Ageing Res Rev 17: 435-447, 2016.

14. Wei Q, Lei R and Hu G: Roles of miR-182 in sensory organ development and cancer. Thorac Cancer 6: 2-9, 2015.

15. Shi Z, Johnson JJ, Jiang R, Liu Y and Stack MS: Decrease of miR-146a is associated with the aggressiveness of human oral squamous cell carcinoma. Arch Oral Biol 60: 1416-1427, 2015.

16. Liu X, Xu B, Li S, Zhang B, Mao P, Qian B, Guo L and Ni P. Association of SNPs in miR-146a, miR-196a2, and miR-499 with the risk of endometrial/ovarian cancer. Acta Biochim Biophys Sin (Shanghai) 47: 564-566, 2015.
17. Stückrath I, Rack B, Janni W, Jäger B, Pantel K and Schwarzenbach H: Aberrant plasma levels of circulating miR-16, miR-107, miR-130a and miR-146a are associated with lymph node metastasis and receptor status of breast cancer patients. Oncotarget 6: 13387-13401, 2015.

18. Zhang WJ, Wang H, Tong QX, Jie SH, Yang DL and Peng C: nvolvement of TLR2-MyD88 in abnormal expression of miR-146a in peripheral blood monocytes of patients with chronic hepatitis C. J Huazhong Univ Sci Technolog Med Sci 35: 266-271, 2015.

19. Chen L, Dai YM, Ji CB, Yang L, Shi CM, Xu GF, Pang LX, Huang FY, Zhang CM and Guo XR: MiR-146b is a regulator of human visceral preadipocyte proliferation and differentiation and its expression is altered in human obesity. Mol Cell Endocrinol 393: 65-74, 2014.

20. Liu J, Xu J, Li H, Sun C, Yu L, Li Y, Shi C and Zhou X: miR-146b-5p functions as a tumor suppressor by targeting TRAF6 and predicts the prognosis of human gliomas. J Cell Physiol 231: 328-335, 2015. 